INPLASY

PROTOCOL

To cite: Fu et al. Tanshinone for acute cerebral infarction: $A$ protocol of systematic review and meta-analysis. Inplasy protocol 202190017. doi: 10.37766/inplasy2021.9.0017

Received: 06 September 2021

Published: 06 September 2021

Corresponding author: Zhaolin Fu

1901025@ccmu.edu.cn

Author Affiliation:

Capital Medical University

Support: None.

Review Stage at time of this submission: The review has not yet started.

Conflicts of interest:

None declared.

\section{Tanshinone for acute cerebral infarction: A protocol of systematic review and meta-analysis}

Fu, Z1; Sun, Z2; Yan, Z3; Shan, S4; Zhang, X5; Wang, M6.

Review question / Objective: Tanshinone administration combined traditonal treatments improve therapeutic effect for acute Cerebral Infarction. P: patients with Cerebral Infarction; I: Tanshinone administration combined traditional treatments; C: traditional treatments such as Aspirin, clopidogrel and edaravone; O: Main outcome measures: total clinical efficacy rate; Neurological deficit score(NIHSS); Worsening situation. Secondary outcome indicators: Inflammatory indicators [hSCRP, IL-6, TNF-a]; Adverse drug reactions. S: Randomized Controlled Trial.

Condition being studied: Acute cerebral infarction, also known as acute ischemic stroke, is caused by a variety of reasons caused by vascular occlusion or stenosis of which supply blood disorder, cause local brain tissue hypoxia and ischemia, the ensuing brain degeneration or necrosis and neural function damage, is the most common type of stroke, accounts for about $60 \% \sim 80 \%$ of the total cases.

INPLASY registration number: This protocol was registered with the International Platform of Registered Systematic Review and Meta-Analysis Protocols (INPLASY) on 06 September 2021 and was last updated on 06 September 2021 (registration number INPLASY202190017).

\section{INTRODUCTION}

Review question / Objective: Tanshinone administration combined traditonal treatments improve therapeutic effect for acute Cerebral Infarction. P: patients with Cerebral Infarction; I: Tanshinone administration combined traditional treatments; C: traditional treatments such as Aspirin, clopidogrel and edaravone; 0 : Main outcome measures: total clinical efficacy rate; Neurological deficit score(NIHSS); Worsening situation. Secondary outcome indicators: Inflammatory indicators [hS-CRP, IL-6, 
TNF-a]; Adverse drug reactions. S: Randomized Controlled Trial.

Rationale: Two researchers will independently research eligible randomized controlled trials in 8 repositories: PubMed, Web of Science, EMBASE, China National Knowledge Infrastructure (CNKI), China Biomedical Literature Service System (sinomed), Database of Chinese sci-tech journals (VIP), Wanfang Data as well as Cochrane Library, from their onset to present. The languages will constitute either English or Chinese, and we will carry out article selection, data mining, and conduct an evaluation of the risk of bias by the Cochrane tool of risk of bias. All analyses will be conducted by using the Cochrane Review Manager software (RevMan 5.4).

Condition being studied: Acute cerebral infarction, also known as acute ischemic stroke, is caused by a variety of reasons caused by vascular occlusion or stenosis of which supply blood disorder, cause local brain tissue hypoxia and ischemia, the ensuing brain degeneration or necrosis and neural function damage, is the most common type of stroke, accounts for about $60 \% \sim 80 \%$ of the total cases.

\section{METHODS}

Participant or population: Acute cerebral infarction patients.

Intervention: Tanshinone administration combined traditional treatments.

Comparator: Tanshinone with other conventional pharmacotherapy to treat acute cerebral infarction.

Study designs to be included: Randomized controlled trials (RCTs) of tanshinone or an intergration of tanshinone with other conventional pharmacotherapy to treat acute cerebral infarction.

Eligibility criteria: Randomized controlled Trials (RCTs) of tanshinone ii A sodium sulfonate injection in the conventional treatment of acute cerebral infarction with or without double blind test.Exclusion criteriaResearch involving any of the following can be excluded: (1) Non-clinical research (animal experiments, clinical review, case analysis);(2) There were studies on other traditional Chinese medicine treatment methods such as acupuncture and moxibustion in the observation group or control group;(3) Literature with incorrect data, incomplete data and unavailable data;(4) Duplicate literature, literature that cannot be obtained in full text;(5) Studies with unclear efficacy evaluation criteria;(6) Only the abstract can not obtain the full text of the literature.

Information sources: PubMed, Web of Science, EMBASE, China National Knowledge Infrastructure (CNKI), China Biomedical Literature Service System (sinomed), Database of Chinese sci-tech journals (VIP), Wanfang Data, Cochrane Library.

Main outcome(s): Total clinical efficacy rate; Neurological deficit score (NIHSS); Worsening situation.

Additional outcome(s): Inflammatory indicators [hS-CRP, IL-6, TNF-a]; Adverse drug reactions.

Data management: The Cochrane Review Manager (RevMan 5.4) software will be employed in data analyses. Continuous variable will be estimated by standardized mean difference (MD), as well as $95 \%$ confidence intervals (Cls). Moreover, the effect size of dichotomous variables will be estimated by the risk ratio, as well as $95 \%$ Cls. The 12 statistic will be utilized to indicate statistical diversity. When $12<50 \%$, we will using a fixed-effect model, otherwise a random-effect model will be employed.

Quality assessment / Risk of bias analysis: If more than 10 studies are included, we will generate Funnel plots, as well as Egger regression text to analyze publication bias. Two authors will evaluate the quality of evidence in systematic review by utilizing 
the grading of recommendations assessment development and evaluation. The result of the assessment will be divided into high, moderate, low, or very low.

Strategy of data synthesis: Using excel to synthesis data from different writers.

Subgroup analysis: We will conduct the subgroup analysis to determine considerable heterogeneity as per study information, patient's features, type of inventing measures, and outcome indicators.

Sensitivity analysis: The sensitivity inspection will be conducted to inspect the robustness of the study. We will eliminate low qualities articles one by one to inspect the reliability of this meta-analysis' results.

Language: Only works in Chinese or English will be included.

Country(ies) involved: China.

Keywords: Tanshinone IIA sulfonate injection; traditional chinese medicine; Acute cerebral infarction; Meta-analysis acute cerebral infarction tanshinone.

Contributions of each author:

Author 1 - Zhaolin Fu.

Author 2 - Ziyi Sun.

Author 3 - Shuyi Shan.

Author 4 - Ziming Yan.

Author 5 - Xiaorui Zhang.

Author 6 - Mengtao Wang. 